\title{
Occasional cooperative breeding in birds and the robustness of comparative analyses concerning the evolution of cooperative breeding
}

\author{
Michael Griesser ${ }^{1,2^{*}}$ (D) and Toshitaka N. Suzuki ${ }^{2}$
}

\begin{abstract}
Cooperative breeding is a widespread and intense form of cooperation, in which individuals help raise offspring that are not their own. This behaviour is particularly well studied in birds, using both long-term and comparative studies that have provided insights into the evolution of reproductive altruism. In most cooperatively breeding species, helpers are offspring that remain with their parents beyond independency and help in the raising of younger siblings. However, many cooperatively breeding species are poorly studied, and in 152 species, this behaviour only has been observed infrequently (i.e., occasional cooperative breeding). Here we argue that the parental care mode of these 152 species needs to be treated with caution, as factors associated with occasional cooperative breeding may differ from those associated with "regular" cooperative breeding. In most cooperatively breeding species, helpers provide alloparental care at the nests of their parents or close relatives; however, only in one occasionally cooperatively breeding species do offspring remain into the next breeding season with their parents. Accordingly, different factors are likely to be associated with regular and occasional cooperative breeding. The latter behaviour resembles interspecific feeding (i.e., individuals feed offspring of another species), which occurs when birds lose their brood and begin feeding at a nearby nest, or when birds mistakenly feed at another nest. Thus, we advise researchers to exclude occasional cooperative breeders in comparative analyses until their status is clarified, or to categorize them separately or according to the typically observed parental care mode. This approach will increase the robustness of comparative analyses and thereby improve our understanding of factors that drive the evolution of cooperative breeding.
\end{abstract}

Keywords: Cooperative breeding, Alloparental care, Comparative studies, Interspecific feeding, Incidental observations

\section{Introduction}

Cooperation among individuals occurs at all levels of biological organisation [1] and understanding the factors that select for cooperation is a fundamental goal of evolutionary biology. Darwin perceived that reproductive cooperation, such as sterile casts in eusocial insects, represented a challenge to his theory of natural selection [2]. Over the past 50 years, research has shown that cooperation often occurs among related individuals $[1,3]$, providing opportunities

\footnotetext{
* Correspondence: michael.griesser@uzh.ch

'Department of Anthropology, University of Zurich, Winterthurerstrasse 190, 8057 Zurich, Switzerland

${ }^{2}$ Department of Evolutionary Studies of Biosystems, SOKENDAI (The Graduate

University for Advanced Studies), Kanagawa, Japan
}

for kin selected fitness benefits [4]. One intensive form of cooperation is alloparental care [1,5-7], in which individuals help raise the offspring of others while often foregoing their own reproduction $[8,9]$. This behaviour has evolved in all major vertebrate lineages and is particularly well investigated in birds, using large-scale comparative studies [10-14].

Earlier comparative studies have investigated the association between eco-climatic and life-history factors and the occurrence of cooperative breeding, yielding sometimes contradictory findings [10-13]. While some studies have suggested that cooperative breeding is associated with stable climatic conditions and saturated habitats $[10,12,15]$, other studies have indicated that 
it is associated with unpredictable climatic conditions $[11,16,17]$. These contradictory findings may in part reflect heterogeneity in the quality of data on the parental care mode of birds. To consider this uncertainty, we categorize species in which cooperative breeding has been observed infrequently as "occasional cooperative breeders" ( $N=152$ species, representing $15 \%$ of both cooperatively and occasionally cooperatively breeding species [18, 19]; supplementary material Additional file 1: Table S1). Observe that we categorized the Darwin finches Geospiza scandens and G. fortis as occasional cooperative breeders, based on a detailed study on helping at the nest in these two species [19] (see below). However, the question of how to categorize the mode of parental care in occasionally cooperatively breeding species remains open, and small numbers of observations may not be sufficient to categorize these species [20].

Here, we review the current understanding of the routes to cooperative breeding, and summarize previously published studies of the family structure of occasional cooperative breeders. We propose that occasional cooperative breeding shows parallels with interspecific feeding (i.e., individuals feed offspring of another species), and thus should not be categorized together with "regular" cooperatively breeding species in comparative studies.

\section{Routes to cooperative breeding}

In nearly all cooperatively breeding species (i.e., $93 \%$ $[18,21,22])$, helpers are offspring that remain associated with their parents until the next breeding season and provide alloparental care at the nest of their parents or close relatives $[9,21,22]$. Field studies have demonstrated that helpers can gain both direct and indirect, kin-selected fitness benefits from providing alloparental care $[3,22,23]$. In some cooperatively breeding species, however, helpers are not related to breeders, but these individuals often have a share in reproduction, the species breeds polygynously or polyandrously (such as ani Crotphasgus sp.), or helpers queue for a breeding position [5, 22]. Thus, this route to cooperative breeding is most likely facilitated by direct fitness benefits [23]. Both of these phenomena occur regularly within populations, but the number of pairs that receive alloparental care can vary between 0 and $100 \%$ depending on the species and annual conditions $[5,6]$. Also, individuals may express a high flexibility in their parental care contributions in some species and frequently switch roles between breeders and helpers even within a breeding season [24]. Finally, three individuals have been observed feeding at the same nest in few instances in 152 species (labelled occasional cooperative breeding [18]), but the factors selecting for this behaviour remain unclear.

\section{Occasional cooperative breeding}

By definition, occasional cooperative breeding occurs rarely, and is thus difficult to investigate and remains poorly understood. This behaviour may occur commonly in some species, but be overlooked in species that are poorly investigated. However, while most cooperatively breeding species are not well studied, (i.e., cooperatively breeding species have a mode of two independent Zoological Record entries; data obtained from [25]; Fig. 1), many occasionally cooperatively breeding species in fact are well studied (mode of independent Zoological Record entries of occasional cooperative breeder $=15 ; N=69$ species have more than 200 independent Zoological Record entries; Spearman rank correlation: $P<0.001$; Fig. 1). For example, occasional cooperative breeding has been

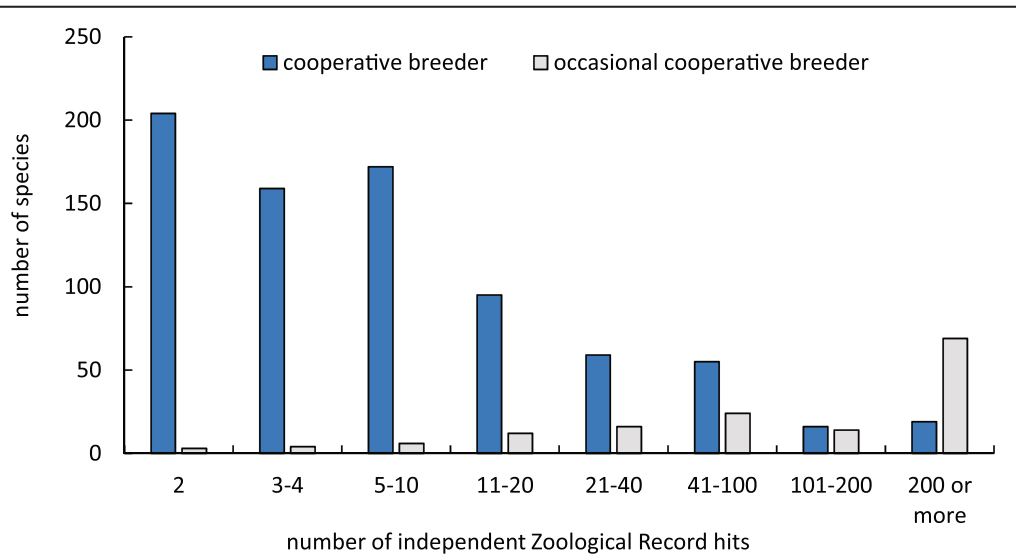

Fig. 1 Distribution of the number of independent Zoological Record entries for cooperatively breeding species, including species that are suspected cooperative breeders (data available for $N=782$ out of 864 species), and occasionally cooperatively breeding species (data available for $N=146$ out of 152 species). Most cooperatively breeding species have only two independent Zoological Record entries, while most occasionally cooperatively breeding species are well studied 
observed in model species, including mute swan Cygnus olor, common guillemot Uria aalge, blue tit Parus caeruleus, white stork Ciconia ciconia, common tern Sterna hirundo or house sparrow Passer domesticus. In these species, "helpers" are most likely unrelated to the breeders, since the offspring do not remain with their parents into the next breeding season, but disperse earlier (see Additional file 2: Table S2 for details) [21, 26-28]. Only in one occasional cooperative breeder, the Verreaux's eagle owl Bubo lacteus, offspring may remain up for to 2 years in the parental territory [29], and thus live in family groups in a manner similar to that of most cooperatively breeding species.

A single study on Darwin finches Geospiza scandens and G. fortis described occasional cooperative breeding in these species in detail [19]. Eleven unpaired adult males were observed to provide alloparental care during a severe drought. However, the helpers were not related to the breeding pair, and many helpers bred earlier on in their lives, excluding that they helped to gain breeding experience. Moreover, none of the females that received help paired up with a helper later on in their lives, and helpers did not seem to gain access to reproduction. Rather, the authors suggested that the alloparental care reflected "misdirected" parental care [19, 30, 31].

\section{Occasional cooperative breeding and interspecific feeding}

Occasional cooperative breeding shows parallels with interspecific feeding (i.e., individuals feed offspring of other species [32]; Figure 2). In both cases, individuals do not feed related offspring, and both behaviours occur rarely. Interspecific feeding has been observed in 51 species (Additional file 2: Table S2; excluding cases where individuals from two species share a nest or egg dumping leads to mixed broods). It has been suggested that the main reason behind interspecific feeding is the loss of the individual's own brood, leading to those individuals to begin feeding at a nearby nest of another species [30, 32, 33]. Alternatively, parents may have their own nest but accidentally feed at another nest due to the close proximity of the other nest, or in response to the begging calls of nestlings [20,32].

Interspecific feeding has been documented in a wide range of bird species (Table 1, Additional file 2: Table S2; see also [32]), suggesting that individuals may feed the young of conspecifics (i.e., engage in occasional cooperative breeding) for the same reasons as individuals engage in interspecific feeding [19]. Indeed, ten species are known to engage both in occasional cooperative breeding and interspecific feeding (i.e., $19.6 \%$ of all occasional cooperative breeders; Additional file 1: Table S1, Additional file 2: Table S2). Interspecific feeding and occasional cooperative breeding, however, differ in one very critical point: the former is easy to recognize and categorize in the field, but a single observation of three individuals feeding at a nest does not allow categorization. It became quickly evident that the Japanese great tit is not a cooperatively breeding species, but one that may engage in interspecific feeding (Figure 2). If the Japanese great tit male had fed at another nest of Japanese great tits, however, we may have classified this observation as regular or occasional cooperative breeding. The generally used definition of cooperative breeding (an individual helps in the raising of offspring that are not their own, often while foregoing their own reproduction $[5,6,8,9])$ does not differentiate whether the behaviour occurs within species (i.e. cooperative breeding), or across species (i.e. interspecific feeding). Thus, we propose to adjust the definition of cooperative breeding to: an individual that helps in the raising of conspecific offspring that are not their own, often while foregoing their own reproduction.

\section{Occasional cooperative breeding and the evolution of regular cooperative breeding}

Historically, observations of occasional cooperative breeding have fuelled a debate on the factors favouring the evolution of cooperative breeding [20, 34]. It has been suggested that the initial evolution of alloparental care may be a non-adaptive response to the begging of nestlings [31]. While this behaviour may provide a first step towards cooperative breeding [34], a number of arguments have been put forward regarding why the behaviour of helpers is modified by natural selection, thus making the behaviour adaptive [34]. Physiological studies showed that helpers in cooperative breeders express higher levels of prolactin than individuals that do not help, and that the prolactin level correlates with the helping effort [35, 36]. Moreover, helpers seem to adjust their feeding effort depending on the need of the nestlings [23, 37-40]. Finally, comparative data have shown that helping behaviour is higher in species in which helping at the nest provides a greater fitness benefit, and helpers in species that exhibit extra-pair mating have a higher degree of kin discrimination [4].

\section{Conclusions}

Interspecific feeding and occasional cooperative breeding have intrigued researchers for decades [30] and stimulated a critical assessment of cooperative breeding [20,34]. Nevertheless, fitness consequences of both interspecific feeding and occasional cooperative breeding remain unstudied due to the rarity of their occurrence [32]. It thus remains difficult to draw firm conclusions as to whether these behaviours are non-adaptive, or provide fitness benefits to the actor. Cooperative breeding has been shown to be adaptive, helpers respond to the actual need of offspring [37], and most helpers provide care at nests of relatives, offering kin selected fitness 
Table 1 Taxonomic overview on the family level over the parental care mode of occasional cooperative breeders and of 9659 bird species, the number of species showing interspecific feeding, the proportion of cooperatively breeding species within all families, and the number of species in each taxonomic family; CB = cooperative breeder (including both family living cooperative breeders and species with unrelated helpers), FAM = family living species, NO-FAM = non-family living species, UN = unknown parental care mode

\begin{tabular}{|c|c|c|c|c|c|c|c|c|c|c|}
\hline \multirow[t]{2}{*}{ Taxonomic family } & \multicolumn{3}{|c|}{$\begin{array}{l}\text { Parental care mode of occasional } \\
\text { CB species }\end{array}$} & \multicolumn{4}{|c|}{$\begin{array}{l}\text { Inferred parental care mode } \\
\text { of all species }\end{array}$} & \multirow[t]{2}{*}{$\begin{array}{l}\mathrm{N} \text { species engaging } \\
\text { in interspecific feeding }\end{array}$} & \multirow[t]{2}{*}{$\begin{array}{l}\text { Proportion } \\
\text { CB species }\end{array}$} & \multirow[t]{2}{*}{$\begin{array}{l}\text { Total number } \\
\text { of species }\end{array}$} \\
\hline & FAM & NO-FAM & UN & $\mathrm{CB}$ & FAM & NO-FAM & UN & & & \\
\hline Acanthisittidae & 0 & 0 & 0 & 1 & 0 & 1 & 0 & 0 & 0.50 & 2 \\
\hline Acanthizidae & 0 & 0 & 0 & 31 & 15 & 10 & 8 & 0 & 0.48 & 64 \\
\hline Accipitridae & 3 & 11 & 6 & 15 & 30 & 53 & 139 & 1 & 0.06 & 237 \\
\hline Aegithalidae & 0 & 0 & 0 & 5 & 0 & 5 & 0 & 1 & 0.50 & 10 \\
\hline Aegithinidae & 0 & 0 & 0 & 0 & 0 & 0 & 4 & 0 & 0.00 & 4 \\
\hline Aegothelidae & 0 & 0 & 0 & 0 & 0 & 1 & 7 & 0 & 0.00 & 8 \\
\hline Alaudidae & 0 & 2 & 2 & 2 & 6 & 35 & 47 & 0 & 0.02 & 90 \\
\hline Alcedinidae & 0 & 2 & 1 & 15 & 2 & 13 & 65 & 0 & 0.16 & 95 \\
\hline Alcidae & 0 & 1 & 0 & 0 & 0 & 18 & 4 & 0 & 0.00 & 22 \\
\hline Anatidae & 4 & 0 & 0 & 0 & 32 & 61 & 61 & 0 & 0.00 & 154 \\
\hline Anhimidae & 0 & 0 & 0 & 0 & 1 & 2 & 0 & 0 & 0.00 & 3 \\
\hline Anhingidae & 0 & 0 & 0 & 0 & 0 & 4 & 0 & 0 & 0.00 & 4 \\
\hline Anseranatidae & 0 & 0 & 0 & 1 & 0 & 0 & 0 & 0 & 1.00 & 1 \\
\hline Apodidae & 0 & 0 & 0 & 12 & 0 & 24 & 62 & 0 & 0.12 & 98 \\
\hline Apterygidae & 0 & 0 & 0 & 1 & 0 & 3 & 1 & 0 & 0.20 & 5 \\
\hline Aramidae & 0 & 0 & 0 & 0 & 1 & 0 & 0 & 0 & 0.00 & 1 \\
\hline Ardeidae & 0 & 0 & 0 & 0 & 5 & 42 & 15 & 0 & 0.00 & 62 \\
\hline Artamidae & 0 & 0 & 0 & 9 & 0 & 2 & 0 & 0 & 0.82 & 11 \\
\hline Atrichornithidae & 0 & 0 & 0 & 0 & 1 & 1 & 0 & 0 & 0.00 & 2 \\
\hline Balaenicipitidae & 0 & 0 & 0 & 0 & 0 & 1 & 0 & 0 & 0.00 & 1 \\
\hline Bombycillidae & 0 & 0 & 0 & 0 & 0 & 4 & 4 & 0 & 0.00 & 8 \\
\hline Brachypteraciidae & 0 & 0 & 0 & 0 & 1 & 0 & 4 & 0 & 0.00 & 5 \\
\hline Bucconidae & 0 & 0 & 0 & 5 & 0 & 4 & 24 & 0 & 0.15 & 33 \\
\hline Bucerotidae & 1 & 0 & 0 & 21 & 8 & 12 & 13 & 0 & 0.39 & 54 \\
\hline Bucorvidae & 0 & 0 & 0 & 2 & 0 & 0 & 0 & 0 & 1.00 & 2 \\
\hline Burhinidae & 0 & 0 & 0 & 0 & 2 & 4 & 3 & 0 & 0.00 & 9 \\
\hline Callaeatidae & 0 & 0 & 0 & 0 & 0 & 2 & 0 & 0 & 0.00 & 2 \\
\hline Campephagidae & 1 & 0 & 0 & 5 & 5 & 19 & 55 & 0 & 0.06 & 84 \\
\hline Caprimulgidae & 0 & 0 & 1 & 0 & 4 & 10 & 71 & 0 & 0.00 & 85 \\
\hline Cardinalidae & 0 & 1 & 0 & 13 & 6 & 21 & 18 & 2 & 0.22 & 58 \\
\hline Cariamidae & 0 & 0 & 0 & 0 & 2 & 0 & 0 & 0 & 0.00 & 2 \\
\hline Casuariidae & 0 & 0 & 0 & 0 & 1 & 2 & 0 & 0 & 0.00 & 3 \\
\hline Cathartidae & 0 & 0 & 0 & 0 & 3 & 1 & 3 & 0 & 0.00 & 7 \\
\hline Certhiidae & 0 & 0 & 0 & 0 & 2 & 4 & 1 & 0 & 0.00 & 7 \\
\hline Charadriidae & 0 & 0 & 2 & 1 & 8 & 33 & 23 & 0 & 0.02 & 65 \\
\hline Chionidae & 0 & 0 & 0 & 0 & 1 & 1 & 1 & 0 & 0.00 & 3 \\
\hline Chloropseidae & 0 & 0 & 0 & 0 & 0 & 2 & 6 & 0 & 0.00 & 8 \\
\hline Ciconiidae & 0 & 0 & 2 & 0 & 2 & 9 & 8 & 0 & 0.00 & 19 \\
\hline Cinclidae & 0 & 0 & 0 & 0 & 0 & 2 & 3 & 0 & 0.00 & 5 \\
\hline
\end{tabular}


Table 1 Taxonomic overview on the family level over the parental care mode of occasional cooperative breeders and of 9659 bird species, the number of species showing interspecific feeding, the proportion of cooperatively breeding species within all families, and the number of species in each taxonomic family; $C B=$ cooperative breeder (including both family living cooperative breeders and species with unrelated helpers), FAM = family living species, NO-FAM = non-family living species, UN = unknown parental care mode (Continued)

\begin{tabular}{|c|c|c|c|c|c|c|c|c|c|c|}
\hline \multirow[t]{2}{*}{ Taxonomic family } & \multicolumn{3}{|c|}{$\begin{array}{l}\text { Parental care mode of occasional } \\
\text { CB species }\end{array}$} & \multicolumn{4}{|c|}{$\begin{array}{l}\text { Inferred parental care mode } \\
\text { of all species }\end{array}$} & \multirow[t]{2}{*}{$\begin{array}{l}\mathrm{N} \text { species engaging } \\
\text { in interspecific feeding }\end{array}$} & \multirow[t]{2}{*}{$\begin{array}{l}\text { Proportion } \\
\text { CB species }\end{array}$} & \multirow[t]{2}{*}{$\begin{array}{l}\text { Total number } \\
\text { of species }\end{array}$} \\
\hline & $\overline{\text { FAM }}$ & NO-FAM & UN & $\overline{\mathrm{CB}}$ & FAM & NO-FAM & UN & & & \\
\hline Cinclosomatidae & 0 & 0 & 0 & 3 & 0 & 0 & 2 & 0 & 0.60 & 5 \\
\hline Cisticolidae & 0 & 0 & 0 & 14 & 48 & 27 & 29 & 1 & 0.12 & 118 \\
\hline Climacteridae & 0 & 0 & 0 & 5 & 1 & 1 & 0 & 0 & 0.71 & 7 \\
\hline Cnemophilidae & 0 & 0 & 0 & 0 & 0 & 3 & 0 & 0 & 0.00 & 3 \\
\hline Coerebidae & 0 & 0 & 0 & 0 & 0 & 0 & 1 & 0 & 0.00 & 1 \\
\hline Coliidae & 0 & 0 & 0 & 6 & 0 & 0 & 0 & 0 & 1.00 & 6 \\
\hline Colluricinclidae & 0 & 0 & 0 & 6 & 0 & 0 & 7 & 0 & 0.46 & 13 \\
\hline Columbidae & 0 & 0 & 0 & 0 & 3 & 276 & 25 & 0 & 0.00 & 304 \\
\hline Conopophagidae & 0 & 0 & 0 & 0 & 0 & 8 & 0 & 0 & 0.00 & 8 \\
\hline Coraciidae & 0 & 0 & 1 & 1 & 0 & 3 & 8 & 0 & 0.08 & 12 \\
\hline Corcoracidae & 0 & 0 & 0 & 2 & 0 & 0 & 0 & 0 & 1.00 & 2 \\
\hline Corvidae & 1 & 0 & 0 & 48 & 35 & 31 & 5 & 0 & 0.40 & 119 \\
\hline Cotingidae & 0 & 0 & 0 & 1 & 5 & 60 & 28 & 0 & 0.01 & 94 \\
\hline Cracidae & 1 & 0 & 0 & 0 & 38 & 6 & 6 & 0 & 0.00 & 50 \\
\hline Cracticidae & 0 & 0 & 0 & 7 & 0 & 1 & 4 & 0 & 0.58 & 12 \\
\hline Cuculidae & 0 & 2 & 1 & 4 & 4 & 87 & 47 & 0 & 0.03 & 142 \\
\hline Dasyornithidae & 0 & 0 & 0 & 0 & 0 & 3 & 0 & 0 & 0.00 & 3 \\
\hline Dendrocolaptidae & 0 & 0 & 0 & 0 & 5 & 7 & 34 & 0 & 0.00 & 46 \\
\hline Dicaeidae & 0 & 0 & 0 & 0 & 4 & 14 & 26 & 0 & 0.00 & 44 \\
\hline Dicruridae & 0 & 0 & 0 & 1 & 4 & 7 & 12 & 0 & 0.04 & 24 \\
\hline Diomedeidae & 0 & 0 & 0 & 1 & 0 & 13 & 0 & 0 & 0.07 & 14 \\
\hline Dromadidae & 0 & 0 & 0 & 0 & 0 & 1 & 0 & 0 & 0.00 & 1 \\
\hline Dromaiidae & 0 & 0 & 0 & 0 & 1 & 0 & 0 & 0 & 0.00 & 1 \\
\hline Dulidae & 0 & 0 & 0 & 1 & 0 & 0 & 0 & 0 & 1.00 & 1 \\
\hline Emberizidae & 0 & 5 & 0 & 7 & 40 & 200 & 85 & 6 & 0.02 & 332 \\
\hline Estrildidae & 1 & 0 & 0 & 0 & 26 & 89 & 23 & 0 & 0.00 & 138 \\
\hline Eupetidae & 0 & 0 & 0 & 0 & 1 & 1 & 7 & 0 & 0.00 & 9 \\
\hline Eurylaimidae & 0 & 0 & 0 & 3 & 0 & 3 & 9 & 0 & 0.20 & 15 \\
\hline Eurypygidae & 0 & 0 & 0 & 0 & 0 & 1 & 0 & 0 & 0.00 & 1 \\
\hline Falconidae & 0 & 5 & 1 & 15 & 3 & 14 & 31 & 0 & 0.24 & 63 \\
\hline Falcunculidae & 0 & 0 & 0 & 1 & 0 & 0 & 1 & 0 & 0.50 & 2 \\
\hline Formicariidae & 0 & 0 & 0 & 0 & 1 & 1 & 59 & 0 & 0.00 & 61 \\
\hline Fregatidae & 0 & 0 & 0 & 0 & 2 & 3 & 0 & 0 & 0.00 & 5 \\
\hline Fringillidae & 1 & 6 & 1 & 3 & 30 & 89 & 37 & 3 & 0.02 & 159 \\
\hline Furnariidae & 0 & 0 & 0 & 8 & 2 & 16 & 209 & 0 & 0.03 & 235 \\
\hline Galbulidae & 0 & 0 & 0 & 18 & 0 & 0 & 0 & 0 & 1.00 & 18 \\
\hline Gaviidae & 0 & 0 & 0 & 0 & 0 & 5 & 0 & 1 & 0.00 & 5 \\
\hline Glareolidae & 0 & 0 & 0 & 0 & 3 & 7 & 7 & 0 & 0.00 & 17 \\
\hline
\end{tabular}


Table 1 Taxonomic overview on the family level over the parental care mode of occasional cooperative breeders and of 9659 bird species, the number of species showing interspecific feeding, the proportion of cooperatively breeding species within all families, and the number of species in each taxonomic family; $C B=$ cooperative breeder (including both family living cooperative breeders and species with unrelated helpers), FAM = family living species, NO-FAM = non-family living species, UN = unknown parental care mode (Continued)

\begin{tabular}{|c|c|c|c|c|c|c|c|c|c|c|}
\hline \multirow[t]{2}{*}{ Taxonomic family } & \multicolumn{3}{|c|}{$\begin{array}{l}\text { Parental care mode of occasional } \\
\text { CB species }\end{array}$} & \multicolumn{4}{|c|}{$\begin{array}{l}\text { Inferred parental care mode } \\
\text { of all species }\end{array}$} & \multirow[t]{2}{*}{$\begin{array}{l}N \text { species engaging } \\
\text { in interspecific feeding }\end{array}$} & \multirow[t]{2}{*}{$\begin{array}{l}\text { Proportion } \\
\text { CB species }\end{array}$} & \multirow[t]{2}{*}{$\begin{array}{l}\text { Total number } \\
\text { of species }\end{array}$} \\
\hline & FAM & NO-FAM & UN & $\overline{C B}$ & FAM & NO-FAM & UN & & & \\
\hline Gruidae & 0 & 0 & 0 & 0 & 13 & 1 & 1 & 0 & 0.00 & 15 \\
\hline Haematopodidae & 1 & 0 & 0 & 1 & 5 & 3 & 1 & 0 & 0.10 & 10 \\
\hline Heliornithidae & 0 & 0 & 0 & 0 & 0 & 2 & 1 & 0 & 0.00 & 3 \\
\hline Hemiprocnidae & 0 & 0 & 0 & 0 & 0 & 0 & 4 & 0 & 0.00 & 4 \\
\hline Hirundinidae & 0 & 6 & 0 & 0 & 3 & 65 & 19 & 1 & 0.00 & 87 \\
\hline Hydrobatidae & 0 & 0 & 0 & 0 & 0 & 10 & 10 & 0 & 0.00 & 20 \\
\hline Ibidorhynchidae & 0 & 0 & 0 & 0 & 0 & 0 & 1 & 0 & 0.00 & 1 \\
\hline Icteridae & 0 & 1 & 0 & 11 & 26 & 62 & 12 & 0 & 0.10 & 111 \\
\hline Indicatoridae & 0 & 0 & 0 & 0 & 0 & 17 & 0 & 0 & 0.00 & 17 \\
\hline Irenidae & 0 & 0 & 0 & 0 & 0 & 1 & 1 & 0 & 0.00 & 2 \\
\hline Jacanidae & 0 & 0 & 0 & 0 & 2 & 2 & 4 & 0 & 0.00 & 8 \\
\hline Laniidae & 0 & 1 & 0 & 6 & 8 & 16 & 1 & 0 & 0.19 & 31 \\
\hline Laridae & 3 & 0 & 1 & 0 & 18 & 28 & 51 & 1 & 0.00 & 97 \\
\hline Leptosomidae & 0 & 0 & 0 & 0 & 0 & 1 & 0 & 0 & 0.00 & 1 \\
\hline Machaerirhynchidae & 0 & 0 & 0 & 0 & 0 & 0 & 2 & 0 & 0.00 & 2 \\
\hline Malaconotidae & 1 & 0 & 0 & 7 & 14 & 27 & 6 & 0 & 0.13 & 54 \\
\hline Maluridae & 0 & 0 & 0 & 28 & 0 & 0 & 0 & 0 & 1.00 & 28 \\
\hline Megapodiidae & 0 & 0 & 0 & 0 & 0 & 4 & 15 & 0 & 0.00 & 19 \\
\hline Melanocharitidae & 0 & 0 & 0 & 0 & 1 & 4 & 7 & 0 & 0.00 & 12 \\
\hline Meliphagidae & 1 & 5 & 0 & 21 & 13 & 89 & 54 & 0 & 0.12 & 177 \\
\hline Menuridae & 0 & 0 & 0 & 0 & 2 & 0 & 0 & 0 & 0.00 & 2 \\
\hline Meropidae & 0 & 0 & 0 & 20 & 0 & 0 & 6 & 0 & 0.77 & 26 \\
\hline Mesitornithidae & 0 & 0 & 0 & 2 & 0 & 1 & 0 & 0 & 0.67 & 3 \\
\hline Mimidae & 0 & 0 & 0 & 7 & 8 & 4 & 16 & 2 & 0.20 & 35 \\
\hline Momotidae & 0 & 0 & 0 & 0 & 0 & 0 & 9 & 0 & 0.00 & 9 \\
\hline Monarchidae & 1 & 0 & 0 & 6 & 16 & 23 & 51 & 0 & 0.06 & 96 \\
\hline Motacillidae & 0 & 4 & 1 & 0 & 10 & 32 & 23 & 1 & 0.00 & 65 \\
\hline Muscicapidae & 0 & 7 & 0 & 26 & 24 & 55 & 179 & 3 & 0.09 & 284 \\
\hline Musophagidae & 0 & 0 & 0 & 5 & 1 & 13 & 4 & 0 & 0.22 & 23 \\
\hline Nectariniidae & 0 & 0 & 1 & 4 & 5 & 48 & 67 & 1 & 0.03 & 124 \\
\hline Neosittidae & 0 & 0 & 0 & 2 & 0 & 0 & 0 & 0 & 1.00 & 2 \\
\hline Numididae & 0 & 0 & 0 & 0 & 5 & 1 & 0 & 0 & 0.00 & 6 \\
\hline Nyctibiidae & 0 & 0 & 0 & 0 & 0 & 0 & 7 & 0 & 0.00 & 7 \\
\hline Odontophoridae & 0 & 0 & 0 & 0 & 21 & 10 & 0 & 0 & 0.00 & 31 \\
\hline Opisthocomidae & 0 & 0 & 0 & 1 & 0 & 0 & 0 & 0 & 1.00 & 1 \\
\hline Oriolidae & 0 & 0 & 1 & 2 & 1 & 15 & 12 & 0 & 0.07 & 30 \\
\hline Orthonychidae & 0 & 0 & 0 & 0 & 1 & 1 & 3 & 0 & 0.00 & 5 \\
\hline Otididae & 0 & 0 & 0 & 0 & 10 & 5 & 10 & 0 & 0.00 & 25 \\
\hline
\end{tabular}


Table 1 Taxonomic overview on the family level over the parental care mode of occasional cooperative breeders and of 9659 bird species, the number of species showing interspecific feeding, the proportion of cooperatively breeding species within all families, and the number of species in each taxonomic family; $C B=$ cooperative breeder (including both family living cooperative breeders and species with unrelated helpers), FAM = family living species, NO-FAM = non-family living species, UN = unknown parental care mode (Continued)

\begin{tabular}{|c|c|c|c|c|c|c|c|c|c|c|}
\hline \multirow[t]{2}{*}{ Taxonomic family } & \multicolumn{3}{|c|}{$\begin{array}{l}\text { Parental care mode of occasional } \\
\text { CB species }\end{array}$} & \multicolumn{4}{|c|}{$\begin{array}{l}\text { Inferred parental care mode } \\
\text { of all species }\end{array}$} & \multirow[t]{2}{*}{$\begin{array}{l}N \text { species engaging } \\
\text { in interspecific feeding }\end{array}$} & \multirow[t]{2}{*}{$\begin{array}{l}\text { Proportion } \\
\text { CB species }\end{array}$} & \multirow[t]{2}{*}{$\begin{array}{l}\text { Total number } \\
\text { of species }\end{array}$} \\
\hline & FAM & NO-FAM & UN & $C B$ & FAM & NO-FAM & UN & & & \\
\hline Pachycephalidae & 0 & 0 & 0 & 1 & 1 & 0 & 36 & 0 & 0.03 & 38 \\
\hline Paradisaeidae & 0 & 0 & 0 & 0 & 0 & 6 & 34 & 0 & 0.00 & 40 \\
\hline Pardalotidae & 0 & 0 & 0 & 1 & 0 & 3 & 0 & 0 & 0.25 & 4 \\
\hline Paridae & 1 & 2 & 0 & 14 & 10 & 29 & 4 & 3 & 0.25 & 57 \\
\hline Parulidae & 0 & 4 & 1 & 0 & 7 & 60 & 45 & 2 & 0.00 & 112 \\
\hline Passeridae & 0 & 0 & 0 & 11 & 2 & 24 & 10 & 1 & 0.23 & 47 \\
\hline Pedionomidae & 0 & 0 & 0 & 0 & 1 & 0 & 0 & 0 & 0.00 & 1 \\
\hline Pelecanidae & 0 & 0 & 0 & 0 & 1 & 3 & 4 & 0 & 0.00 & 8 \\
\hline Pelecanoididae & 0 & 0 & 0 & 0 & 0 & 4 & 0 & 0 & 0.00 & 4 \\
\hline Petroicidae & 0 & 1 & 0 & 12 & 0 & 6 & 25 & 1 & 0.28 & 43 \\
\hline Peucedramidae & 0 & 0 & 0 & 0 & 1 & 0 & 0 & 0 & 0.00 & 1 \\
\hline Phaethontidae & 0 & 0 & 0 & 0 & 0 & 3 & 0 & 0 & 0.00 & 3 \\
\hline Phalacrocoracidae & 0 & 1 & 0 & 0 & 1 & 34 & 1 & 0 & 0.00 & 36 \\
\hline Phasianidae & 0 & 0 & 0 & 2 & 70 & 77 & 26 & 0 & 0.01 & 175 \\
\hline Philepittidae & 0 & 0 & 0 & 0 & 0 & 0 & 4 & 0 & 0.00 & 4 \\
\hline Phoenicopteridae & 0 & 0 & 0 & 0 & 0 & 5 & 0 & 0 & 0.00 & 5 \\
\hline Phoeniculidae & 0 & 0 & 0 & 5 & 1 & 2 & 0 & 0 & 0.63 & 8 \\
\hline Picathartidae & 0 & 0 & 0 & 0 & 0 & 2 & 0 & 0 & 0.00 & 2 \\
\hline Picidae & 2 & 2 & 3 & 18 & 44 & 31 & 123 & 3 & 0.08 & 216 \\
\hline Pipridae & 0 & 0 & 0 & 0 & 2 & 24 & 27 & 0 & 0.00 & 53 \\
\hline Pittidae & 0 & 0 & 0 & 0 & 0 & 2 & 29 & 0 & 0.00 & 31 \\
\hline Pityriaseidae & 0 & 0 & 0 & 1 & 0 & 0 & 0 & 0 & 1.00 & 1 \\
\hline Platysteiridae & 0 & 0 & 0 & 13 & 12 & 5 & 0 & 0 & 0.43 & 30 \\
\hline Ploceidae & 0 & 1 & 1 & 9 & 9 & 57 & 31 & 0 & 0.08 & 106 \\
\hline Podargidae & 0 & 0 & 0 & 0 & 2 & 1 & 11 & 0 & 0.00 & 14 \\
\hline Podicipedidae & 5 & 4 & 0 & 0 & 5 & 14 & 0 & 0 & 0.00 & 19 \\
\hline Polioptilidae & 0 & 1 & 0 & 0 & 1 & 4 & 9 & 1 & 0.00 & 14 \\
\hline Pomatostomidae & 0 & 0 & 0 & 5 & 0 & 0 & 0 & 0 & 1.00 & 5 \\
\hline Procellariidae & 0 & 0 & 0 & 0 & 0 & 64 & 12 & 0 & 0.00 & 76 \\
\hline Promeropidae & 0 & 0 & 0 & 0 & 1 & 1 & 0 & 0 & 0.00 & 2 \\
\hline Prunellidae & 0 & 0 & 0 & 13 & 0 & 0 & 0 & 0 & 1.00 & 13 \\
\hline Psittacidae & 1 & 1 & 0 & 22 & 55 & 63 & 206 & 0 & 0.06 & 346 \\
\hline Psophiidae & 0 & 0 & 0 & 3 & 0 & 0 & 0 & 0 & 1.00 & 3 \\
\hline Pteroclididae & 0 & 0 & 0 & 0 & 0 & 16 & 0 & 0 & 0.00 & 16 \\
\hline Ptilonorhynchidae & 0 & 0 & 0 & 0 & 1 & 11 & 8 & 0 & 0.00 & 20 \\
\hline Pycnonotidae & 0 & 0 & 0 & 21 & 38 & 39 & 35 & 0 & 0.16 & 133 \\
\hline Rallidae & 0 & 1 & 0 & 19 & 6 & 14 & 93 & 0 & 0.14 & 132 \\
\hline
\end{tabular}


Table 1 Taxonomic overview on the family level over the parental care mode of occasional cooperative breeders and of 9659 bird species, the number of species showing interspecific feeding, the proportion of cooperatively breeding species within all families, and the number of species in each taxonomic family; $C B=$ cooperative breeder (including both family living cooperative breeders and species with unrelated helpers), FAM = family living species, NO-FAM = non-family living species, UN = unknown parental care mode (Continued)

\begin{tabular}{|c|c|c|c|c|c|c|c|c|c|c|}
\hline \multirow[t]{2}{*}{ Taxonomic family } & \multicolumn{3}{|c|}{$\begin{array}{l}\text { Parental care mode of occasional } \\
\text { CB species }\end{array}$} & \multicolumn{4}{|c|}{$\begin{array}{l}\text { Inferred parental care mode } \\
\text { of all species }\end{array}$} & \multirow[t]{2}{*}{$\begin{array}{l}\mathrm{N} \text { species engaging } \\
\text { in interspecific feeding }\end{array}$} & \multirow[t]{2}{*}{$\begin{array}{l}\text { Proportion } \\
\text { CB species }\end{array}$} & \multirow[t]{2}{*}{$\begin{array}{l}\text { Total number } \\
\text { of species }\end{array}$} \\
\hline & FAM & NO-FAM & UN & $\overline{C B}$ & FAM & NO-FAM & UN & & & \\
\hline Ramphastidae & 0 & 0 & 0 & 38 & 12 & 21 & 52 & 0 & 0.31 & 123 \\
\hline Recurvirostridae & 0 & 0 & 0 & 0 & 4 & 1 & 4 & 0 & 0.00 & 9 \\
\hline Reguliidae & 0 & 0 & 0 & 0 & 0 & 5 & 1 & 0 & 0.00 & 6 \\
\hline Remizidae & 0 & 0 & 0 & 7 & 0 & 4 & 1 & 0 & 0.58 & 12 \\
\hline Rhabdornithidae & 0 & 0 & 0 & 0 & 0 & 3 & 0 & 0 & 0.00 & 3 \\
\hline Rheidae & 0 & 0 & 0 & 1 & 1 & 0 & 0 & 0 & 0.50 & 2 \\
\hline Rhinocryptidae & 0 & 0 & 0 & 0 & 0 & 1 & 28 & 0 & 0.00 & 29 \\
\hline Rhipiduridae & 0 & 0 & 1 & 0 & 1 & 2 & 39 & 0 & 0.00 & 42 \\
\hline Rhynochetidae & 0 & 0 & 0 & 1 & 0 & 0 & 0 & 0 & 1.00 & 1 \\
\hline Rostratulidae & 0 & 0 & 0 & 0 & 1 & 1 & 0 & 0 & 0.00 & 2 \\
\hline Sapayoaidae & 0 & 0 & 0 & 0 & 0 & 1 & 0 & 0 & 0.00 & 1 \\
\hline Scolopacidae & 0 & 0 & 0 & 0 & 1 & 33 & 53 & 0 & 0.00 & 87 \\
\hline Scopidae & 0 & 0 & 0 & 1 & 0 & 0 & 0 & 0 & 1.00 & 1 \\
\hline Sittidae & 0 & 0 & 0 & 2 & 5 & 11 & 7 & 2 & 0.08 & 25 \\
\hline Spheniscidae & 0 & 1 & 0 & 0 & 0 & 17 & 0 & 1 & 0.00 & 17 \\
\hline Steatornithidae & 0 & 0 & 0 & 0 & 0 & 1 & 0 & 0 & 0.00 & 1 \\
\hline Stercorariidae & 0 & 1 & 0 & 2 & 0 & 4 & 2 & 0 & 0.25 & 8 \\
\hline Strigidae & 2 & 3 & 0 & 1 & 14 & 27 & 111 & 1 & 0.01 & 153 \\
\hline Struthionidae & 0 & 0 & 0 & 0 & 1 & 0 & 0 & 0 & 0.00 & 1 \\
\hline Sturnidae & 1 & 1 & 0 & 18 & 8 & 77 & 6 & 1 & 0.17 & 109 \\
\hline Sulidae & 0 & 0 & 0 & 0 & 1 & 8 & 1 & 0 & 0.00 & 10 \\
\hline Sylviidae & 0 & 1 & 1 & 21 & 16 & 50 & 177 & 0 & 0.08 & 264 \\
\hline Thamnophilidae & 0 & 0 & 0 & 2 & 143 & 5 & 46 & 0 & 0.01 & 196 \\
\hline Thinocoridae & 0 & 0 & 0 & 0 & 0 & 2 & 2 & 0 & 0.00 & 4 \\
\hline Thraupidae & 0 & 0 & 0 & 32 & 74 & 110 & 51 & 0 & 0.12 & 267 \\
\hline Threskiornithidae & 0 & 0 & 0 & 0 & 3 & 14 & 15 & 0 & 0.00 & 32 \\
\hline Timaliidae & 0 & 0 & 0 & 81 & 18 & 130 & 54 & 0 & 0.29 & 283 \\
\hline Tinamidae & 0 & 0 & 0 & 0 & 0 & 7 & 39 & 0 & 0.00 & 46 \\
\hline Todidae & 0 & 0 & 0 & 5 & 0 & 0 & 0 & 0 & 1.00 & 5 \\
\hline Trochilidae & 0 & 0 & 0 & 0 & 0 & 310 & 8 & 0 & 0.00 & 318 \\
\hline Troglodytidae & 3 & 0 & 0 & 17 & 15 & 9 & 35 & 3 & 0.22 & 76 \\
\hline Trogonidae & 0 & 0 & 0 & 0 & 1 & 0 & 38 & 0 & 0.00 & 39 \\
\hline Turdidae & 1 & 1 & 0 & 5 & 13 & 115 & 32 & 5 & 0.03 & 165 \\
\hline Turnicidae & 0 & 0 & 0 & 0 & 3 & 10 & 3 & 0 & 0.00 & 16 \\
\hline Tyrannidae & 1 & 0 & 0 & 6 & 37 & 56 & 293 & 3 & 0.02 & 392 \\
\hline Tytonidae & 0 & 0 & 0 & 0 & 0 & 6 & 10 & 0 & 0.00 & 16 \\
\hline
\end{tabular}


Table 1 Taxonomic overview on the family level over the parental care mode of occasional cooperative breeders and of 9659 bird species, the number of species showing interspecific feeding, the proportion of cooperatively breeding species within all families, and the number of species in each taxonomic family; $C B=$ cooperative breeder (including both family living cooperative breeders and species with unrelated helpers), FAM = family living species, NO-FAM = non-family living species, UN = unknown parental care mode (Continued)

\begin{tabular}{|c|c|c|c|c|c|c|c|c|c|c|}
\hline \multirow[t]{2}{*}{ Taxonomic family } & \multicolumn{3}{|c|}{$\begin{array}{l}\text { Parental care mode of occasional } \\
\text { CB species }\end{array}$} & \multicolumn{4}{|c|}{$\begin{array}{l}\text { Inferred parental care mode } \\
\text { of all species }\end{array}$} & \multirow[t]{2}{*}{$\begin{array}{l}\mathrm{N} \text { species engaging } \\
\text { in interspecific feeding }\end{array}$} & \multirow[t]{2}{*}{$\begin{array}{l}\text { Proportion } \\
\text { CB species }\end{array}$} & \multirow[t]{2}{*}{$\begin{array}{l}\text { Total number } \\
\text { of species }\end{array}$} \\
\hline & FAM & NO-FAM & UN & $\overline{C B}$ & FAM & NO-FAM & UN & & & \\
\hline Upupidae & 0 & 0 & 0 & 1 & 0 & 0 & 0 & 0 & 1.00 & 1 \\
\hline Urocynchramidae & 0 & 0 & 0 & 0 & 0 & 0 & 1 & 0 & 0.00 & 1 \\
\hline Vangidae & 0 & 0 & 0 & 4 & 3 & 5 & 8 & 0 & 0.20 & 20 \\
\hline Viduidae & 0 & 0 & 0 & 0 & 0 & 17 & 0 & 0 & 0.00 & 17 \\
\hline Vireonidae & 0 & 0 & 0 & 0 & 5 & 15 & 31 & 0 & 0.00 & 51 \\
\hline Zosteropidae & 0 & 1 & 0 & 6 & 5 & 49 & 34 & 0 & 0.06 & 94 \\
\hline Total & 37 & 86 & 29 & 864 & 1257 & 3654 & 3884 & 51 & 0.09 & 9659 \\
\hline
\end{tabular}

Families with more than $25 \%$ cooperative breeders are highlighted in bold. Taxonomy follows Jetz et al. [44]. See main text and [21] for definitions of the parental care mode. The inferred parental care mode follows Cockburn [18], updated based on the Handbook of the Birds of the World [45]. Observe that we categorized the Darwin finches Geospiza scandens and G. fortis as occasional cooperative breeders, based on a detailed study on helping at the nest in these two species [19]

benefits [22]. Moreover, family living is a stepping stone for the evolution of cooperative breeding [21, 26, 41, 42], but most occasionally cooperatively breeding species do not live in family groups (Additional file 1: Table S1).

Further studies are needed to assess whether "helpers" in occasional cooperative breeders gain direct fitness benefits from doing so (e.g. by having a share in reproduction through extra pair mating or egg dumping). Until the factors that facilitate cooperative breeding in these species are better understood, it may be misleading to categorize such species as cooperative breeders in comparative studies. Thus, we advise excluding these 152 occasional cooperative breeders from analyses of cooperative breeding until their mode of parental care is critically assessed, or to categorize
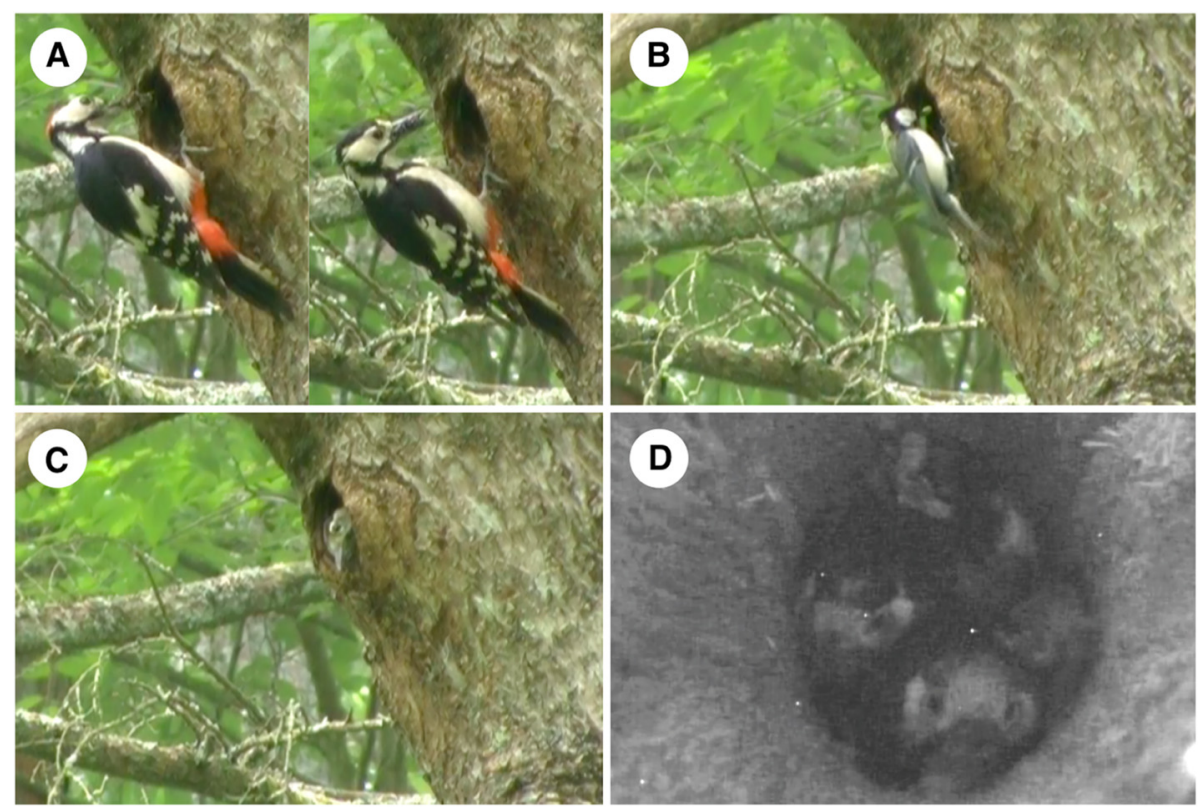

Fig. 2 An example of interspecific feeding. During fieldwork on Japanese great tits Parus minor in Karuizawa, Nagano Prefecture, Japan (36.33'138' N, 138.56'260" E) 15-18 June 2015, we observed a nest of a great spotted woodpecker Dendrocopos major where in addition to the parents. (a), a Japanese great tit male fed the young woodpeckers (b). Based on video recordings, the feeding rate of the parents was much lower than the feeding rate by the great tit (feeding rates: male parent: 5.2/hr, female parent: $4.1 / \mathrm{hr}$, great tit male: 17.2/hr, total time assessed: 14.5 hrs). Inspection with an infrared camera confirmed that the cavity contained four great spotted woodpecker nestlings but no great tit nestlings (c-d). Moreover, the woodpeckers often displaced the male Japanese great tit from the cavity 
them separately or according to the typically observed parental care mode. This approach will increase the robustness of comparative analyses and thereby improve our understanding of factors that drive the evolution of cooperative breeding.

\section{Ethical statements}

Our field work was approved by the Animal Care and Use Committees at SOKENDAI (The Graduate University for Advanced Studies) and the Forestry Agency of Japan. Our work adhered to the Guidelines for the Use of Animals in Research of the Animal Behavior Society/ Association for the Study of Animal Behaviour.

\section{Additional files}

Additional file 1: List of species that are categorized as occasional cooperatively breeding species. (DOCX $45 \mathrm{~kb}$ )

Additional file 2: List of species that have been observed to engage in misdirected parental care (i.e., feeding young at the nest of another species), excluding cases where species shared nests. (DOCX $30 \mathrm{~kb}$ )

\section{Competing interests}

The authors declare that they have no competing interests.

\section{Authors' contributions}

Data collection: MG, TS; data analyses: MG, TS; writing manuscript: MG, TS. Both authors read and approved the final manuscript.

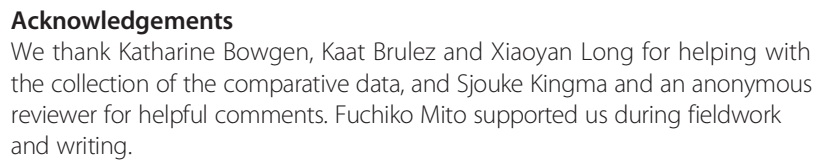

We thank Katharine Bowgen, Kaat Brulez and Xiaoyan Long for helping with the collection of the comparative data, and Sjouke Kingma and an anonymous reviewer for helpful comments. Fuchiko Mito supported us during fieldwork and writing.

\section{Data sharing}

We adhere to the data sharing policies outlined in Mills et al. [43].

Received: 2 October 2015 Accepted: 22 February 2016

Published online: 28 March 2016

\section{References}

1. Bourke AFG. Principles of social evolution. Oxford series in ecology and evolution. Oxford: Oxford University Press; 2011.

2. Darwin C. On the origin of species. London: John Murray; 1859.

3. Hamilton WD. Genetical evolution of social behaviour I. J Theor Biol. 1964;7(1):1-16.

4. Cornwallis CK, West SA, Griffin AS. Routes to indirect fitness in cooperatively breeding vertebrates: kin discrimination and limited dispersal. J Evol Biol. 2009;22(12):2445-57.

5. Cockburn A. Evolution of helping behavior in cooperatively breeding birds. Annu Rev Ecol Syst. 1998;29:141-77.

6. Stacey PB, Koenig WD. Cooperative breeding in birds. Cambridge: Cambridge University Press; 1990

7. Solomon NG, French JA. Cooperative breeding in mammals. Cambridge: Cambridge University Press; 1997.

8. Koenig WD, Dickinson JL. Ecology and evolution of cooperative breeding in birds. Cambridge: Cambridge University Press; 2004.

9. Hatchwell BJ. The evolution of cooperative breeding in birds: kinship, dispersal and life history. Philos Trans R Soc Lond B Biol Sci. 2009;364(1533): 3217-27. doi:10.1098/rstb.2009.0109.

10. Arnold KE, Owens IPF. Cooperative breeding in birds: a comparative test of the life history hypothesis. Proc R Soc B-Biol Sci. 1998;265(1398):739-45.
11. Jetz W, Rubenstein DR. Environmental uncertainty and the global biogeography of cooperative breeding in birds. Curr Biol. 2011;21(1):72-8. doi:10.1016/j.cub.2010.11.075

12. Gonzalez J-CT, Sheldon BC, Tobias JA. Environmental stability and the evolution of cooperative breeding in hornbills. Proc R Soc B Biol Sci. 2013;280(1768):20131297.

13. Cornwallis CK, West SA, Davis KE, Griffin AS. Promiscuity and the evolutionary transition to complex societies. Nature. 2010;466(7309):969-72.

14. Downing PA, Cornwallis CK, Griffin AS. Sex, long life and the evolutionary transition to cooperative breeding in birds. Proc R Soc B. 2015;282(1816): 20151663.

15. Emlen ST. The Evolution of helping.1. an ecological constraints model. Am Nat. 1982;119(1):29-39.

16. Rubenstein DR, Lovette IJ. Temporal environmental variability drives the evolution of cooperative breeding in birds. Curr Biol. 2007;17(16):1414-9.

17. Koenig WD, Mumme RL. Population ecology of the cooperative breeding acorn woodpecker. Princeton: Princeton University Press; 1987.

18. Cockburn A. Prevalence of different modes of parental care in birds. Proc $R$ Soc B Biol Sci. 2006;273(1592):1375-83. doi:10.1098/rspb.2005.3458.

19. Price T, Millington S, Grant P. Helping at the nest in Darwin's finches as misdirected parental care. Auk. 1983;100:192-4.

20. Jamieson IG. Behavioral heterochrony and the evolution of birds' helping at the nest: An unselected consequences of communal breeding? Am Nat. 1989;133:394-406

21. Drobniak SM, Wagner G, Mourocq E, Griesser M. Family living: an overlooked but pivotal social system to understand the evolution of cooperative breeding. Behav Ecol. 2015;26(3):805-11. doi:10.1093/beheco/ arv015.

22. Riehl C. Evolutionary routes to non-kin cooperative breeding in birds. Proc $\mathrm{R}$ Soc B Biol Sci. 2013;280(1772):20132245.

23. Dickinson JL, Hatchwell BJ. Fitness consequnces of helping. In: Koenig WD, Dickinson J, editors. Ecology and evolution of cooperative breeding in birds. Cambridge: Cambridge University Press; 2004. p. 48-66.

24. Preston SA, Briskie JV, Burke T, Hatchwell BJ. Genetic analysis reveals diverse kin-directed routes to helping in the rifleman Acanthisitta chloris. Mol Ecol. 2013:22(19):5027-39.

25. Valcu M, Dale J, Griesser M, Nakagawa S, Kempenaers B. Global gradients of avian longevity support the classic evolutionary theory of ageing. Ecography. 2014;37(10):930-8. doi:10.1111/ecog.00929.

26. Covas R, Griesser M. Life history and the evolution of family living in birds. Proc R Soc B Biol Sci. 2007;274(1616):1349-57.

27. Griesser M, Nystrand M, Ekman J. Reduced mortality selects for family cohesion in a social species. Proc R Soc B Biol Sci. 2006;273(1596):1881-6.

28. Griesser M, Barnaby J. Families, a place of loving care and violent conflicts. The role of nepotism, cooperation and competition in the evolution of avian families. In: Columbus F, editor. Behavioral and chemical ecology: new research. New York: Nova; 2010. p. 47-90.

29. Hockey PAR, Dean WJJ, Ryan PG. Roberts birds of Southern Africa. 7th ed. Trustees of the John Voelcker Bird Book Fund: Cape Town; 2005.

30. Skutch AF. Helpers among birds. Condor. 1961;63:198-226.

31. Jamieson I. The unselected hypothesis for the evolution of helpingbehavior - too much or too little emphasis on natural-selection. Am Nat. 1991;138(1):271-82.

32. Shy MM. Interspecific feeding among birds: a review. J Field Ornithology. 1982;53:370-93.

33. Jacot $A$, Reers $H$, Forstmeier $W$. Individual recognition and potential recognition errors in parent-offspring communication. Behav Ecol Sociobiol. 2010;64(10): 1515-25.

34. Emlen ST, Reeve HK, Sherman PW, Wrege PH, Ratnieks FLW, Shellmanreeve J. Adaptive versus nonadaptive explanations of behavior - the case of alloparental helping. Am Nat. 1991;138(1):259-70.

35. Carlson AA, Russell AF, Young AJ, Jordan NR, McNeilly AS, Parlow AF, et al. Elevated prolactin levels immediately precede decisions to babysit by male meerkat helpers. Horm Behav. 2006;50(1):94-100. doi:10.1016/j.yhbeh. 2006.01.009.

36. Schoech SJ, Mumme RL, Wingfield JC. Prolactin and helping behaviour in the cooperatively breeding Florida scrub-jay, Aphelocoma coerulescens. Anim Behav. 1996:52:445-56.

37. Canestrari D, Chiarati E, Marcos JM, Ekman J, Baglione V. Helpers but not breeders adjust provisioning effort to year-round territory resource availability in carrion crows. Anim Behav. 2008;76:943-9. doi:10.1016/j.anbehav.2008.05.013. 
38. Kingma SA, Hall ML, Peters A. Multiple benefits drive helping behavior in a cooperatively breeding bird: an integrated analysis. Am Nat. 2011;177(4): 486-95. doi:10.1086/658989.

39. Nam K-B, Simeoni M, Sharp SP, Hatchwell BJ. Kinship affects investment by helpers in a cooperatively breeding bird. Proc R Soc B Biol Sci. 2010; 277(1698):3299-306. doi:10.1098/rspb.2010.0737.

40. Heinsohn R, Legge S. The cost of helping. Trends Ecol Evol. 1999;14(2):53-7.

41. Ekman J, Dickinson JL, Hatchwell BJ, Griesser M. Delayed dispersal. In: Koenig WD, Dickinson JL, editors. Ecology and evolution of cooperative breeding in birds. Cambridge: Cambridge University Press; 2004.

42. Ekman J, Griesser M. Siberian jays: delayed dispersal in absence of cooperative breeding. In: Koenig WD, Dickinson J, editors. Cooperative breeding in vertebrates: studies of ecology, evolution, and behavior. Cambridge: Cambridge University Press; 2016. p. 6-18.

43. Mills JA, Teplitsky C, Arroyo B, Charmantier A, Becker PH, Birkhead TR, et al. Archiving primary data: solutions for long-term studies. Trends Ecol Evol. 2015:30(10):581-9. doi:10.1016/j.tree.2015.07.006.

44. Jetz W, Thomas GH, Joy JB, Hartmann K, Mooers AO. The global diversity of birds in space and time. Nature. 2012;491(7424):444-8. doi:10.1038/nature11631.

45. Del Hoyo J, Elliot A, Sargatal J, Christie DA. Handbook of the birds of the world. Barcelona: Lynx Editions; 2011.

\section{Submit your next manuscript to BioMed Central} and we will help you at every step:

- We accept pre-submission inquiries

- Our selector tool helps you to find the most relevant journal

- We provide round the clock customer support

- Convenient online submission

- Thorough peer review

- Inclusion in PubMed and all major indexing services

- Maximum visibility for your research

Submit your manuscript at www.biomedcentral.com/submit 